\title{
Radio Astronomical Polarimetry and the Lorentz Group
}

\author{
M. C. Britton \\ Swinburne Centre for Astrophysics and Supercomputing, Swinburne University of Technology, \\ Hawthorn, Victoria 3122, Australia
}

\begin{abstract}
In radio astronomy the polarimetric properties of radiation are often modified during propagation and reception. Effects such as Faraday rotation, receiver cross-talk, and differential amplification act to change the state of polarized radiation. A general description of such transformations is useful for the investigation of these effects and for the interpretation and calibration of polarimetric observations. Such a description is provided by the Lorentz group, which is intimately related to the transformation properties of polarized radiation. In this paper the transformations that commonly arise in radio astronomy are analyzed in the context of this group. This analysis is then used to construct a model for the propagation and reception of radio waves. The implications of this model for radio astronomical polarimetry are discussed.
\end{abstract}

Subject headings: polarization — techniques: polarimetric

\section{Introduction}

In radio astronomy transformations occur during the propagation and reception of radio waves that act to change the state of polarized radiation. Some of these transformations, such as Faraday rotation in the ionosphere or the interstellar medium, arise from propagation effects that may themselves be of astrophysical interest. Others originate from instrumental effects such as differential amplification or receiver cross-talk, and have an adverse effect on polarimetric observations. Realistically many such effects may be present, each having its own time and frequency dependence, and collectively acting to distort measurements of the polarized radiation. The interpretation and calibration of these observations may be quite complex, and it is useful to have a general context in which to describe these transformations.

Linear transformations of fully polarized radiation were first investigated by Jones (1941), who represented transformations of the two-component transverse electric field in terms of $2 \times 2$ complex matrices now called Jones matrices. This analysis was extended to partially polarized radiation by Parrent \& Roman (1960), who used Jones matrices to describe the transformation properties of the coherency matrix. Alternatively, both fully and partially polarized radiation may be described by the Stokes parameters, and their linear transformations may be represented in terms of 4x4 real matrices called Mueller matrices (Mueller 1948). 
In fact these transformations are intimately related to the Lorentz group. This relationship arises from the fact that for a plane propagating wave Maxwell's equations admit two independent solutions, representing the two orthogonal senses of polarization. Thus polarized radiation constitutes a two state system, and the linear transformations of all such systems are described by the Lorentz group. This relationship has long been known in optics, and different aspects have been discussed by many authors (e.g. Barakat 1963, Whitney 1971, Cloude 1986, Pellat-Finet \& Bausset 1992). Recent work has focused on representations of the Jones and Mueller matrices (Opatrny \& Perina 1993, Brown \& Bak 1995, Han, Kim \& Noz 1997). Up to a multiplicative constant the set of Jones matrices constitute the group $\mathrm{SL}(2, \mathrm{C})$, which forms the spin $1 / 2$ representation of the Lorentz group. The corresponding set of Mueller matrices constitutes the group $\mathrm{SO}(3,1)$ and forms the spin 1 representation of the Lorentz group. Finally, the relationship between Jones and Mueller matrices is represented through the 2-1 mapping between these two groups. $\mathrm{SO}(3,1)$.

The formulation of the transformation properties of polarized radiation in terms of Lorentz transformations affords considerable insight, including the interpretation of the Stokes parameters as a Lorentz 4-vector, the classification of transformations of this 4-vector as rotations or boosts, and the existence of a polarimetric analogue to the invariant interval. In this paper these concepts are reviewed in the context of radio astronomical polarimetry. Since both linear and circular bases are widely used in radio astronomy, a basis independent formulation is emphasized. This formalism is then used to construct a model for the propagation and reception of radio waves.

\section{Representations of Polarized Radiation}

Consider the representation of a transverse electromagnetic wave. Such a wave may be described through its two-component transverse electric field vector $\mathcal{E}(t)$. This vector may also represent the electric field in a waveguide, or the voltage in a pair of cables. The vector $\mathcal{E}(t)$ is commonly written in terms of the two-component complex analytic signal $\mathbf{E}(t)$ as $\mathcal{E}(t)=\operatorname{Re}[\mathbf{E}(t) \exp \{i \omega t\}]$. This construction is familiar from both optics (Born \& Wolf 1980) and signal processing (Bracewell 1986).

For fully polarized radiation the analytic signal is independent of time. In this case the relative amplitudes and phases of the two components of $\mathbf{E}$ specify the state of elliptical polarization of the plane wave. For partially polarized radiation $\mathbf{E}(t)$ is time dependent, and measurable properties of the wave may instead be defined through time-averaging. Such an averaging procedure is conveniently treated through the coherency matrix (Wiener 1930, Wolf 1959). This is a 2x2 Hermitian matrix formed from the direct product of the analytic signal, and may be written as $\rho=\left\langle\mathbf{E}(t) \otimes \mathbf{E}^{\dagger}(t)\right\rangle$. Here the angular brackets denote time-averaging. As with any Hermitian matrix, the 
coherency matrix may be written in terms of 4 real quantities $\left(S_{o}, \mathbf{S}\right)$ as

$$
\rho=\left(S_{o} \sigma_{o}+\mathbf{S} \cdot \sigma\right) / 2
$$

where $\sigma_{\mathbf{o}}$ is the $2 \times 2$ identity matrix and $\sigma$ is a 3 -vector whose components are the Pauli spin matrices. These three $2 \times 2$ matrices are traceless and Hermitian with determinant -1 . The 4 parameters $\left(S_{o}, \mathbf{S}\right)$ are simply the mean Stokes parameters of the plane wave (Fano 1954), with $S_{o}$ representing the total intensity.

Let us now introduce a particular basis. The electric field vector may be represented in the Cartesian basis $(\hat{x}, \hat{y}, \hat{z})$, in which $\mathcal{E}(t)=\left(\mathcal{E}_{x}(t), \mathcal{E}_{y}(t)\right)$ is resolved into mutually orthogonal components, each orthogonal to the direction of propagation $z$ of the plane wave. The corresponding analytic signal is $\mathbf{E}(t)=\left(E_{x}(t), E_{y}(t)\right)$. For the 3 -dimensional space of $\mathbf{S}$ the Cartesian basis $(\hat{q}, \hat{u}, \hat{v})$ is used, along with the customary representation of the Pauli matrices

$$
\sigma_{\hat{q}}=\left(\begin{array}{cc}
1 & 0 \\
0 & -1
\end{array}\right) \quad \sigma_{\hat{u}}=\left(\begin{array}{cc}
0 & 1 \\
1 & 0
\end{array}\right) \quad \sigma_{\hat{v}}=\left(\begin{array}{cc}
0 & -i \\
i & 0
\end{array}\right)
$$

To associate the coherency matrix with the Stokes parameters, we write $\rho$ in this basis as

$$
\rho=\left\langle\mathbf{E}(\mathbf{t}) \otimes \mathbf{E}^{\dagger}(\mathbf{t})\right\rangle=\left(\begin{array}{ll}
\left\langle E_{x}^{*}(t) E_{x}(t)\right\rangle & \left\langle E_{x}^{*}(t) E_{y}(t)\right\rangle \\
\left\langle E_{y}^{*}(t) E_{x}(t)\right\rangle & \left\langle E_{y}^{*}(t) E_{y}(t)\right\rangle
\end{array}\right)
$$

From equation 1, the Stokes parameters in this basis become

$$
\begin{array}{ll}
S_{o}=\left\langle E_{x}^{*}(t) E_{x}(t)\right\rangle+\left\langle E_{y}^{*}(t) E_{y}(t)\right\rangle & S_{u}=2 \operatorname{Re}\left[\left\langle E_{x}^{*}(t) E_{y}(t)\right\rangle\right] \\
S_{q}=\left\langle E_{x}^{*}(t) E_{x}(t)\right\rangle-\left\langle E_{y}^{*}(t) E_{y}(t)\right\rangle & S_{v}=2 \operatorname{Im}\left[\left\langle E_{x}^{*}(t) E_{y}(t)\right\rangle\right]
\end{array}
$$

This is simply the usual definition of the Stokes parameters in a linear basis (Born \& Wolf 1980).

\section{Transformation Properties of Polarized Radiation}

Let us now consider the transformation properties of polarized radiation. Attention is restricted to linear, invertible transformations. This excludes the class of projective transformations, which are important in representing perfect polarizing filters.

Similarly, such transformations cannot describe multipath propagation of coherent radiation, such as the focusing or defocusing of radiation by lenses or mirrors. Despite this restriction, the set of linear, invertible transformations encompasses a broad class of physical processes, including single-particle scattering, propagation through anisotropic media, and many transformations arising from instrumental devices. This set may also describe the linear transformations of the voltage signal in two cables, which are known in linear network theory as two-port networks (Ruston \& Bordogna 1966). This equivalence is particularly useful in radio astronomy, where the two 
components of the electric field are converted to voltages by a receiver and then passed through an electronics downconversion chain.

The most general linear transformation of the analytic signal may be written as $\mathbf{E}^{\prime}(t)=\mathbf{t E}(t)$, where the Jones matrix $\mathbf{t}$ is a $2 \times 2$ complex matrix. As the direct product of the analytic signal, the coherency matrix must transform as $\rho^{\prime}=\mathbf{t} \rho \mathbf{t}^{\dagger}$. For invertible transformations a Jones matrix may be written as $\mathbf{t}=\sqrt{\operatorname{det} \mathbf{t}} \mathbf{t}_{N}$, where det $\mathbf{t}$ is the determinant of $\mathbf{t}$ and $\mathbf{t}_{N}$ is a matrix with unit determinant. The set of $2 \times 2$ complex invertible matrices with unit determinant forms the group $\mathrm{SL}(2, \mathrm{C})$, which constitutes the spin 1/2 representation of the Lorentz group.

To investigate the transformation properties of the Stokes parameters, note that the determinant of equation 1 is simply det $\rho=S_{o}^{2}-|S|^{2} \equiv S_{\text {inv }}$ (Barakat 1963). This is just the form of the Lorentz invariant. Under transformation by the Jones matrix $\mathbf{t}$, det $\rho^{\prime}=|\sqrt{\operatorname{det} \mathbf{t}}|^{2} S_{\mathrm{inv}}$, so that this interval is preserved up to a multiplicative constant. The set of transformations that preserve this interval forms the group $\mathrm{SO}(3,1)$, which constitutes the spin 1 representation of the Lorentz group. That is, the Stokes parameters transform as a Lorentz 4-vector, with the total intensity acting as the timelike component and the remaining Stokes parameters acting as the spacelike components. The condition that the total intensity $S_{o}>0$ restricts this 4 -vector to lie within or on the surface of the forward light cone. These two cases correspond to partially polarized $\left(S_{\mathrm{inv}}>0\right)$ or fully polarized $\left(S_{\mathrm{inv}}=0\right)$ radiation, respectively.

The representations of the groups $\mathrm{SL}(2, \mathrm{C})$ and $\mathrm{SO}(3,1)$ are well known in physics, but are not widely used in astronomy. Basis-independent representations of these groups are now reviewed, and are interpreted in the context of polarimetry. This will serve both as an introduction and to establish the notation used in the next section. For similar reviews, see Brown \& Bak (1995) or Tung (1996).

The group $\mathrm{SL}(2, \mathrm{C})$ contains as a subgroup the set of $2 \times 2$ unitary transformations $\mathrm{SU}(2)$. Any such unitary transformation may be parameterized as

$$
\mathbf{r}_{\hat{\mathbf{n}}}(\phi)=e^{(i \sigma \cdot \hat{\mathbf{n}} \phi)}=\sigma_{0} \cos \phi+i \sigma \cdot \hat{\mathbf{n}} \sin \phi
$$

where $\hat{\mathbf{n}}$ is a unit 3 -vector. This is called the axis-angle parameterization of $\mathrm{SU}(2)$. The angle $\phi$ differs from the definition customary in classical and quantum mechanics by a factor of $1 / 2$, but is in agreement with the conventions of optics. Under the transformation of equation $5, \rho \rightarrow \mathbf{r}_{\hat{\mathbf{n}}}(\phi) \rho \mathbf{r}_{\hat{\mathbf{n}}}(-\phi)=\left(\sigma_{o} S_{o}+\sigma \cdot \mathbf{S}^{\prime}\right) / 2$, where

$$
\mathbf{S}^{\prime}=\mathbf{S} \cos 2 \phi+\mathbf{S} \times \hat{\mathbf{n}} \sin 2 \phi+(\hat{\mathbf{n}} \cdot \mathbf{S}) \hat{\mathbf{n}}(1-\cos 2 \phi)
$$

and we have used the relationship $(\sigma \cdot \mathbf{a})(\sigma \cdot \mathbf{b})=\mathbf{a} \cdot \mathbf{b}+i \sigma \cdot(\mathbf{a} \times \mathbf{b})$. But $\mathbf{S}^{\prime}$ is simply the vector resulting from a rotation of $\mathbf{S}$ about an axis $\hat{\mathbf{n}}$ by an angle $2 \phi$ ( $c f$. Goldstein 1980). This reflects the well-known mapping between $\mathrm{SU}(2)$ and the group $\mathrm{SO}(3)$, whose elements form a representation of the rotations of a 3-dimensional vector. The mapping is 2-1, since the two rotations $\mathbf{r}_{\hat{\mathbf{n}}}(\phi)$ and $\mathbf{r}_{\hat{\mathbf{n}}}(\phi+\pi)=-\mathbf{r}_{ \pm \hat{\mathbf{n}}}(\phi)$ result in the 
same vector $\mathbf{S}^{\prime}$. Such rotations preserve the degree of polarization $|\mathbf{S}| / S_{o}$ of the plane wave, and are readily interpreted geometrically in the space of the Poincare sphere in terms of the axis $\hat{\mathbf{n}}$ and angle $2 \phi$ of rotation. Equation 5 may be represented in the basis of equation 2 as

$$
\mathbf{r}_{\hat{\mathbf{n}}}(\phi)=\left(\begin{array}{cc}
\cos \phi+i n_{q} \sin \phi & \left(i n_{u}+n_{v}\right) \sin \phi \\
\left(i n_{u}-n_{v}\right) \sin \phi & \cos \phi-i n_{q} \sin \phi
\end{array}\right)
$$

where $\hat{\mathbf{n}}=\left(n_{\hat{q}}, n_{\hat{u}}, n_{\hat{v}}\right)$. From equation 6 the corresponding rotation of the Stokes parameters $\left(S_{o}, S_{q}, S_{u}, S_{v}\right)$ in this basis is

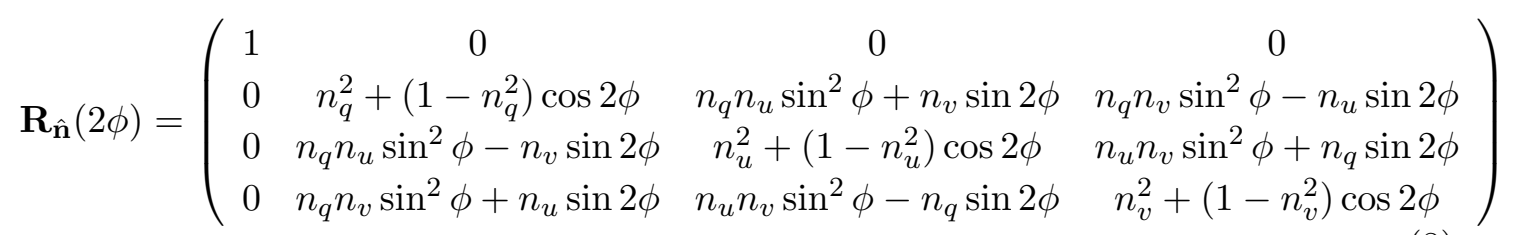

For example, for $\left(n_{\hat{q}}, n_{\hat{u}}, n_{\hat{v}}\right)=(1,0,0)$ equation 8 constitutes the rotation about the $\hat{q}$ axis $\mathbf{R}_{\hat{\mathbf{q}}}(2 \phi)$, which may be interpreted from equation $\mathbf{0}$ as generating a phase delay between the two components of the electric field.

Next let us consider the group $\mathrm{SL}(2, \mathrm{C})$, which has a parameterization similar to its subgroup $\mathrm{SU}(2)$. Any element of the group may be written as $\exp (i \sigma \cdot \hat{\mathbf{n}} \phi+\sigma \cdot \hat{\mathbf{m}} \beta)$, where $\hat{\mathbf{n}}$ and $\hat{\mathbf{m}}$ are unit vectors. In analogy with equation $\mathbf{0}$, let us consider transformations for which $\phi=0$. Such transformations may be written in terms of the Hermitian matrix

$$
\mathbf{b}_{\hat{\mathbf{m}}}(\beta)=\exp (\sigma \cdot \hat{\mathbf{m}} \beta)=\sigma_{0} \cosh \beta+\sigma \cdot \hat{\mathbf{m}} \sinh \beta
$$

Under this transformation the coherency matrix becomes $\rho \rightarrow \mathbf{b}_{\hat{\mathbf{m}}}(\beta) \rho \mathbf{b}_{\hat{\mathbf{m}}}(\beta)=$ $\left(\sigma_{o} S_{o}^{\prime}+\sigma \cdot \mathbf{S}^{\prime}\right) / 2$, where

$$
\begin{gathered}
S_{o}^{\prime}=S_{o} \cosh 2 \beta+\mathbf{S} \cdot \hat{\mathbf{m}} \sinh 2 \beta \\
\mathbf{S}^{\prime}=\mathbf{S}+\left(S_{o} \sinh 2 \beta+2 \mathbf{S} \cdot \hat{\mathbf{m}} \sinh ^{2} \beta\right) \hat{\mathbf{m}}
\end{gathered}
$$

This is simply the result of performing a Lorentz boost on the 4 -vector $S=\left(S_{o}, \mathbf{S}\right)$ along the axis $\hat{\mathbf{m}}$ by a velocity parameter $2 \beta$ ( $c f$. Jackson 1975). As in the general case, such a transformation preserves the invariant interval $S_{\text {inv }}$. Unlike rotations, it does not preserve the degree of polarization $|\mathbf{S}| / S_{o}$ of the plane wave. For example, there exists some transformation that will completely depolarize partially polarized radiation. This is just the analogue of the statement in special relativity that there always exists a reference frame in which two events separated by a timelike interval occur at the same position in space. Equation 9 may be represented in the basis of equation 2 as

$$
\mathbf{b}_{\hat{\mathbf{m}}}(\beta)=\left(\begin{array}{cc}
\cosh \beta+m_{q} \sinh \beta & \left(m_{u}-i m_{v}\right) \sinh \beta \\
\left(m_{u}+i m_{v}\right) \sinh \beta & \cosh \beta-m_{q} \sinh \beta
\end{array}\right)
$$


where $\hat{\mathbf{m}}=\left(m_{\hat{q}}, m_{\hat{u}}, m_{\hat{v}}\right)$. The boost of equation 10 in this basis becomes

$$
\mathbf{B}_{\hat{\mathbf{m}}}(2 \beta)=\left(\begin{array}{cccc}
\cosh 2 \beta & m_{q} \sinh 2 \beta & m_{u} \sinh 2 \beta & m_{v} \sinh 2 \beta \\
m_{q} \sinh 2 \beta & 1+2 m_{q}^{2} \sinh ^{2} \beta & 2 m_{q} m_{u} \sinh ^{2} \beta & 2 m_{q} m_{v} \sinh ^{2} \beta \\
m_{u} \sinh 2 \beta & 2 m_{q} m_{u} \sinh ^{2} \beta & 1+2 m_{u}^{2} \sinh ^{2} \beta & 2 m_{u} m_{v} \sinh ^{2} \beta \\
m_{v} \sinh 2 \beta & 2 m_{q} m_{v} \sinh ^{2} \beta & 2 m_{u} m_{v} \sinh ^{2} \beta & 1+2 m_{v}^{2} \sinh ^{2} \beta
\end{array}\right)
$$

The rotations of equations 0 and 8 and boosts of equation 11 and 12 constitute a subset of the Lorentz transformations that suffices for the radio astronomical applications presented in the next section.

Finally, systems consisting of multiple physical processes are readily modelled through successive application of the above transformations. Of particular use in the analysis of such composite systems are the commutation relations

$$
\left[\mathbf{R}_{\hat{\mathbf{n}}}(\alpha) \mathbf{R}_{\hat{\mathbf{n}}}(\beta)\right]=\left[\mathbf{R}_{\hat{\mathbf{n}}}(\alpha) \mathbf{B}_{\hat{\mathbf{n}}}(\beta)\right]=\left[\mathbf{B}_{\hat{\mathbf{n}}}(\alpha) \mathbf{B}_{\hat{\mathbf{n}}}(\beta)\right]=0
$$

As we shall see in the next section, these relationships are useful in determining whether the transformations that arise from different physical processes or instrumental elements commute with one another.

\section{The Propagation and Reception of Radio Waves}

Let us examine some practical situations in which linear, invertible transformations of polarized radiation arise. One such example is a rotation of the electric field vector about the direction of propagation. This transformation may represent a rotation of a physical device with respect to the plane wave. Another example arises from Faraday rotation, which occurs when radio waves propagate through a magnetized plasma. Such a transformation may be written as $\mathbf{r}_{\hat{v}}(\phi)$. Equivalently, the transformation of the Stokes parameters is $\mathbf{R}_{\hat{v}}(2 \phi)$. A similar transformation generates a phase delay between the two components of $\mathbf{E}$, and may be written as the rotation $\mathbf{r}_{\hat{q}}(\psi)$. In optics a physical device that induces such a phase delay is called a compensator. This transformation arises in electronics when two signals traverse different cable lengths. Both $\mathbf{r}_{\hat{v}}(\phi)$ and $\mathbf{r}_{\hat{q}}(\psi)$ are unitary, and preserve the degree of polarization $|\mathbf{S}| / S_{o}$ and the invariant interval $S_{\text {inv }}$.

Differential amplification or attenuation of the components of $\mathbf{E}$ provide examples of a non-unitary transformation. Consider the transformation

$$
\mathbf{g}=\left(\begin{array}{cc}
g_{a} & 0 \\
0 & g_{b}
\end{array}\right)
$$

We may write this as $\mathbf{g}=\sqrt{g_{a} g_{b}} \mathbf{b}_{\hat{q}}(\beta)$, where $\beta=\ln \left(g_{b} / g_{a}\right)$. The Stokes parameters transform as $g_{a} g_{b} \mathbf{B}_{\hat{q}}(2 \beta)$. Note that this transformation does not preserve $|\mathbf{S}| / S_{o}$, and preserves $S_{\text {inv }}$ only up to the factor $g_{a} g_{b}$. 
Next consider two orthogonal elliptically polarized waves with axial ratio $\tan \chi$ and orientation $\theta$ (Chandrasekhar 1960).

$$
e_{a}=\left(\begin{array}{c}
\cos \theta \cos \chi-i \sin \theta \sin \chi \\
-\sin \theta \cos \chi-i \cos \theta \sin \chi
\end{array}\right) \quad e_{b}=\left(\begin{array}{c}
\sin \theta \cos \chi-i \cos \theta \sin \chi \\
\cos \theta \cos \chi+i \sin \theta \sin \chi
\end{array}\right)
$$

We may perform a change of basis by forming a matrix $\mathbf{s}$ with rows $e_{a}^{\dagger}$ and $e_{b}^{\dagger}$. This matrix may be factored as $\mathbf{s}(\theta, \chi)=\mathbf{r}_{\hat{u}}(\chi) \mathbf{r}_{\hat{v}}(\theta)$. A transformation from a linear to a circular basis occurs for $\chi=\pi / 4$, and in the case where $\theta=\pi / 4$ results simply in a cyclic permutation of the indices $(\hat{q}, \hat{u}, \hat{v}) \rightarrow(\hat{v}, \hat{q}, \hat{u})$ in equation 2 . The transformation $\mathbf{s}(\theta, \chi)$ may also be regarded as representing a receiver with two receptors sensitive to orthogonal forms of elliptical radiation. The process of reception constitutes a projection of $\mathbf{E}$ onto the receptors of the receiver, which is represented by matrix multiplication of $\mathbf{E}$ by $\mathbf{s}(\theta, \chi)$. Clearly the choice of a rotation about the $\hat{u}$ axis followed by one about the $\hat{v}$ axis is not unique. One alternative specification of elliptically polarized radiation is given in terms of an orientation and phase delay as $\mathbf{r}_{\hat{v}}(\theta) \mathbf{r}_{\hat{q}}(\phi)$. In optics this transformation is accomplished through a device known as a Babinet compensator (Born \& Wolf 1980).

Now let us consider a receiver sensitive to two forms of elliptical polarization that are not necessarily orthogonal. This situation may arise in practice from imperfections in the construction of a receiver (Conway \& Kronberg 1969, Stinebring et al. 1984). The transformation may be written as

$$
\mathbf{c}=\left(\begin{array}{cc}
\cos \theta_{a} \cos \chi_{a}+i \sin \theta_{a} \sin \chi_{a} & -\sin \theta_{a} \cos \chi_{a}+i \cos \theta_{a} \sin \chi_{a} \\
\sin \theta_{b} \cos \chi_{b}+i \cos \theta_{b} \sin \chi_{b} & \cos \theta_{b} \cos \chi_{b}-i \sin \theta_{b} \sin \chi_{b}
\end{array}\right)
$$

where the two probes of the receiver are sensitive to elliptical radiation with axial ratios $\chi_{a}, \chi_{b}$ and orientations $\theta_{a}, \theta_{b}$. For the case $\theta_{a}=\theta_{b}$ and $\chi_{a}=\chi_{b}$ equation 16 simplifies to the unitary transformation $\mathbf{s}(\theta, \chi)$. In the general case such a transformation does not conserve energy. With the definitions

$$
\begin{array}{ll}
\sigma_{\theta}=\theta_{a}+\theta_{b} & \sigma_{\chi}=\chi_{a}+\chi_{b} \\
\delta_{\theta}=\theta_{a}-\theta_{b} & \delta_{\chi}=\chi_{a}-\chi_{b}
\end{array}
$$

we may write $\mathbf{c}=\mathbf{c}^{\prime} \mathbf{r}_{\hat{v}}\left(\sigma_{\theta} / 2\right)$, where

$$
\mathbf{c}^{\prime}=\left(\begin{array}{cc}
\cos \left(\sigma_{\chi} / 2+\delta_{\chi} / 2\right) \cos \delta_{\theta} / 2+ & -\cos \left(\sigma_{\chi} / 2+\delta_{\chi} / 2\right) \sin \delta_{\theta} / 2+ \\
i \sin \left(\sigma_{\chi} / 2+\delta_{\chi} / 2\right) \sin \delta_{\theta} / 2 & i \sin \left(\sigma_{\chi} / 2+\delta_{\chi} / 2\right) \cos \delta_{\theta} / 2 \\
-\cos \left(\sigma_{\chi} / 2-\delta_{\chi} / 2\right) \sin \delta_{\theta} / 2+ & \cos \left(\sigma_{\chi} / 2-\delta_{\chi} / 2\right) \cos \delta_{\theta} / 2+ \\
i \sin \left(\sigma_{\chi} / 2-\delta_{\chi} / 2\right) \cos \delta_{\theta} / 2 & i \sin \left(\sigma_{\chi} / 2-\delta_{\chi} / 2\right) \sin \delta_{\theta} / 2
\end{array}\right)
$$

For a nearly orthogonal receiver with receptors sensitive to linearly polarized radiation, this matrix may be written to first order in these parameters as 
$\mathbf{c}^{\prime}=\mathbf{b}_{\hat{u}}^{(1)}\left(\delta_{\theta} / 2\right) \mathbf{b}_{\hat{v}}^{(1)}\left(\delta_{\chi} / 2\right) \mathbf{r}_{\hat{u}}^{(1)}\left(\sigma_{\chi} / 2\right)$. Here the superscript (1) indicates that these transformations are first order in their arguments.

These examples may be combined to form a model for the propagation and reception of radio waves.

$$
\mathbf{t}=\sqrt{g_{a} g_{b}} \mathbf{b}_{\hat{q}}(\beta) \mathbf{r}_{\hat{q}}\left(\Phi_{I}\right) \mathbf{b}_{\hat{u}}^{(1)}\left(\delta_{\theta} / 2\right) \mathbf{b}_{\hat{v}}^{(1)}\left(\delta_{\chi} / 2\right) \mathbf{r}_{\hat{u}}^{(1)}\left(\sigma_{\chi} / 2\right) \mathbf{r}_{\hat{v}}\left(\sigma_{\theta} / 2\right) \mathbf{r}_{\hat{v}}(\zeta) \mathbf{r}_{\hat{v}}\left(\Phi_{\text {iono }}\right) \mathbf{r}_{\hat{v}}\left(\Phi_{\text {ISM }}\right)
$$

where $\Phi_{\text {iono }}$ and $\Phi_{\text {ISM }}$ are the angles arising from Faraday rotation in the ionosphere and the interstellar medium, respectively, $\zeta$ is the angle between the frame of the receiver and that of the sky, and $\Phi_{I}$ is the instrumental phase delay arising from differing electronic pathlengths. The equivalent transformation law for the Stokes parameters is simply obtained from equation 19.

$S^{\prime}=g_{a} g_{b} \mathbf{B}_{\hat{q}}(2 \beta) \mathbf{R}_{\hat{q}}\left(2 \Phi_{I}\right) \mathbf{B}_{\hat{u}}^{(1)}\left(\delta_{\theta}\right) \mathbf{B}_{\hat{v}}^{(1)}\left(\delta_{\chi}\right) \mathbf{R}_{\hat{u}}^{(1)}\left(\sigma_{\chi}\right) \mathbf{R}_{\hat{v}}\left(\sigma_{\theta}\right) \mathbf{R}_{\hat{v}}(2 \zeta) \mathbf{R}_{\hat{v}}\left(2 \Phi_{\text {iono }}\right) \mathbf{R}_{\hat{v}}\left(2 \Phi_{\text {ISM }}\right) S$

The analysis of this model is simplified through the commutation relations in equation 13. Amplifications and phase delays in the downconversion chain represent boosts and rotations with respect to the $\hat{q}$ axis, so it is easy to see that the order of amplifiers and relative electronics delays does not matter. Terms from individual components may simply be collected into the overall parameters $\beta$ and $\Phi_{I}$. Similarly, rotations about the same axis commute, and rotations about the $\hat{v}$ axis by the angles $\zeta$, $\Phi_{\text {iono }}$, and $\Phi_{\text {ISM }}$ all have the same signature. Note that each of the parameters in this model may have its own time and frequency dependence. For example, $\Phi_{\text {iono }}$ fluctuates in time as the ionospheric column density changes, and scales as $\nu^{-2}$ from the cold plasma dispersion relation. Finally, calibration of a polarimetric observation is accomplished through the inversion of equations 19 or 20. Naturally such an inversion requires a knowledge of the parameters in this model.

\section{Discussion}

The representation of polarimetric transformations in terms of the Lorentz group provide a simple context in which to analyze polarized radiation. This formalism is particularly relevant for the calibration of polarimetric data, and greatly simplifies the discussion from a qualitative standpoint. Propagation or instrumental effects that give rise to the rotations of equation 8 change the polarized component of the radiation $\mathbf{S}$, thus obscuring the properties of the true, emitted light. Those effects that take the form of a boost transformation mix the total intensity $S_{o}$ and the polarized component of the radiation $\mathbf{S}$. Such transformations can have a particularly detrimental effect on polarimetric observations. In many astrophysical applications $S_{o}$ is much larger than $|\mathbf{S}|$, so that even boosts nearly equivalent to the identity matrix may completely corrupt the polarized flux. Observations that aim to detect very small polarized fractions, 
such as the polarized component of the Cosmic Microwave Background radiation or the circularly polarized radio emission from Active Galactic Nuclei, are particularly vulnerable. For applications that require extremely high precision, the mixing of $\mathbf{S}$ into $S_{o}$ can corrupt an observation. One such example has been seen in high-precision pulsar timing, where differential amplification and receiver cross-talk induce time dependent mixing of the pulse profiles, thereby modifying the total intensity profile and systematically shifting the times of arrival (Britton et al., in preparation). For pulsar observations the invariant $S_{\text {inv }}$ proves particularly useful, since an invariant pulse profile may be formed that is independent of propagation and instrumental effects. This invariant profile may then be used for timing or to investigate pulse variability that may be intrinsic to the pulsar.

I thank René Grognard, Richard Manchester, Geoffrey Opat, and Matthew Bailes for useful conversations. I thank the referee for pointing out recent literature on the application of the Lorentz group to optics.

\section{REFERENCES}

Barakat, R. 1963, J. Opt. Soc. Am. 53, 317

Born, M. \& Wolf, E. 1980, "Principles of Optics" (Cambridge: University Press)

Bracewell, R. N. 1986, "The Fourier Transform and its Applications" (New York: McGraw Hill)

Brown, C. S. \& Bak, A. E. 1995, Optical Engineering 34, 1625

Chandrasekhar, S. 1960, "Radiative Transfer" (New York: Dover)

Cloude, S. R. 1986, Optik, 75, 26

Conway, R. G., \& Kronberg, P. P. 1969, MNRAS 142, 11

Fano, U. 1954, Phys. Rev. 93, 121

Goldstien, H. 1980, "Classical Mechanics" (Reading, Massachusetts: Addison-Wesley)

Han, D., Kim, Y. S. \& Noz, M. E. 1997a, Phys. Rev. E, 56, 6065

Jackson, J.D. 1975, "Classical Electrodynamics" (New York: John Wiley \& Sons)

Jones, R. C. 1941, J. Opt. Soc. Am. 31, 488

Mueller, H. 1948, J. Opt. Soc. Am. 38, 661

Opatrny, T. \& Perina, J 1993, Physics Letters A, 181, 199

Parrent, G. B. \& Roman, P. 1960, Nuovo Cimento, 15, 370

Pellat-Finet, P. \& Bausset, M. 1992, Optik, 90, 101 
Ruston, H. \& Bordogna, J. 1966, "Electric Networks: functions, filters, analysis" (New York: McGraw-Hill)

Stinebring, D. R., Cordes, J. M., Rankin, J. M., Weisberg, J. M., \& Boriakoff, V. 1984, ApJS, 55, 247

Whitney, C. 1971, J. Opt. Soc. Am., 61, 1207

Wiener, N. 1930, Acta Math. 55, 118

Wolf, E. 1959, Nuovo Cimento, 13, 1165

Tung, W. K. 1985, "Group Theory in Physics" (Philadelphia: World Scientific) 